\title{
Introduction to Remote Sensing Image Registration
}

\author{
Jacqueline Le Moigne, \\ NASA Goddard Space Flight Center
}

IGARSS 2017 


\section{Problem Description}

- $\quad$ Earth Science studies such as:

- Climate change over multiple time scales

- Predicting crop production

- Monitoring land resources

- Understanding the impact of human activity on major Earth ecosystems

- Addressed by using global and repetitive measurements provided by a wide variety of satellite remote sensing systems

- Multiple-time or simultaneous observations of the same Earth features by different sensors

- Global measurements with remote sensing systems

- Complemented by regional and local measurements using ground and airborne sensors

- Addressed by using global and repetitive measurements provided by a wide variety of satellite remote sensing systems

- Need to correlate and integrate all these complementary data 


\section{NASA Earth Global Measurements}

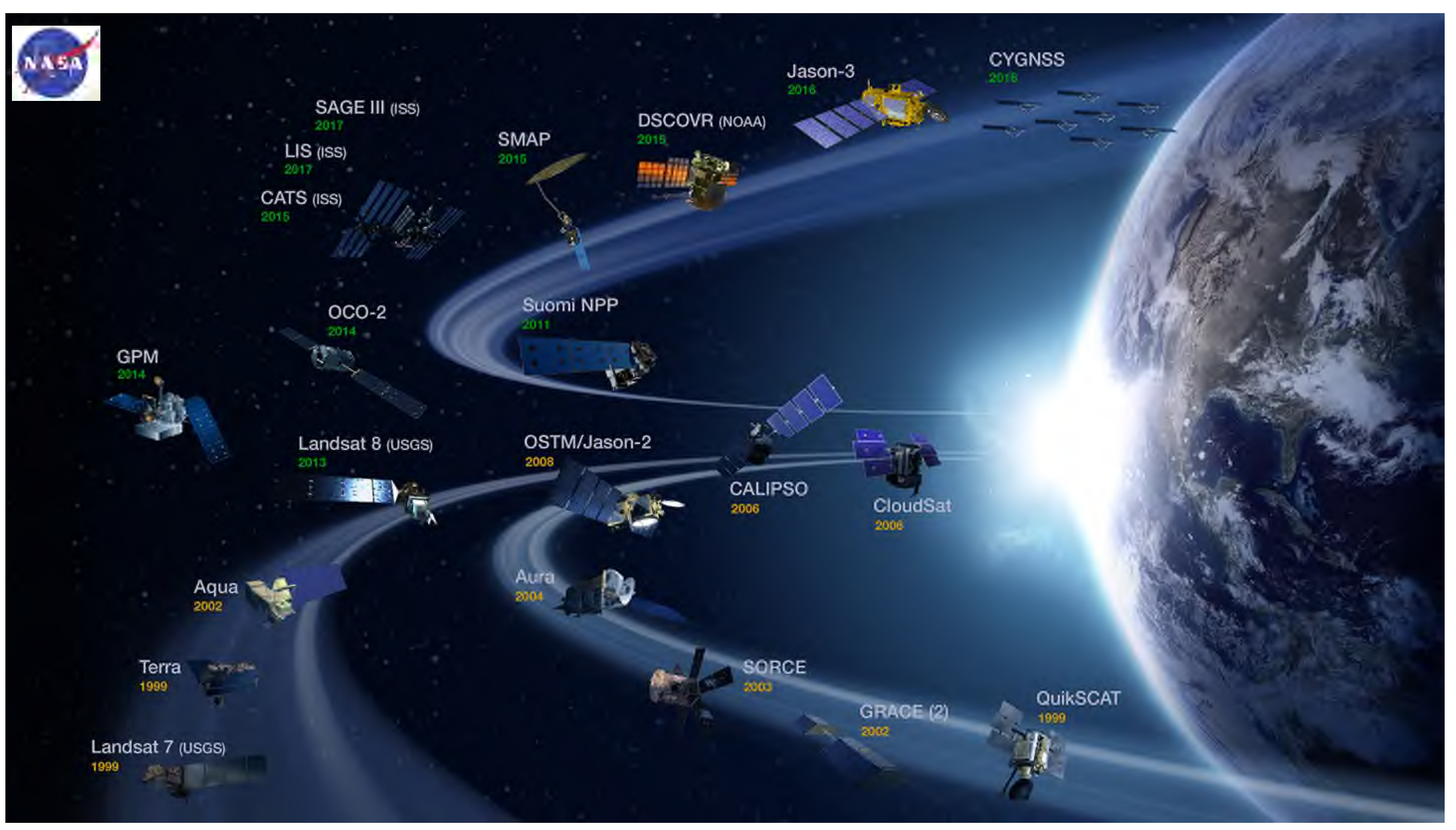




\section{Example of International Measurements}

คลง

GPM Constellation of Satellites

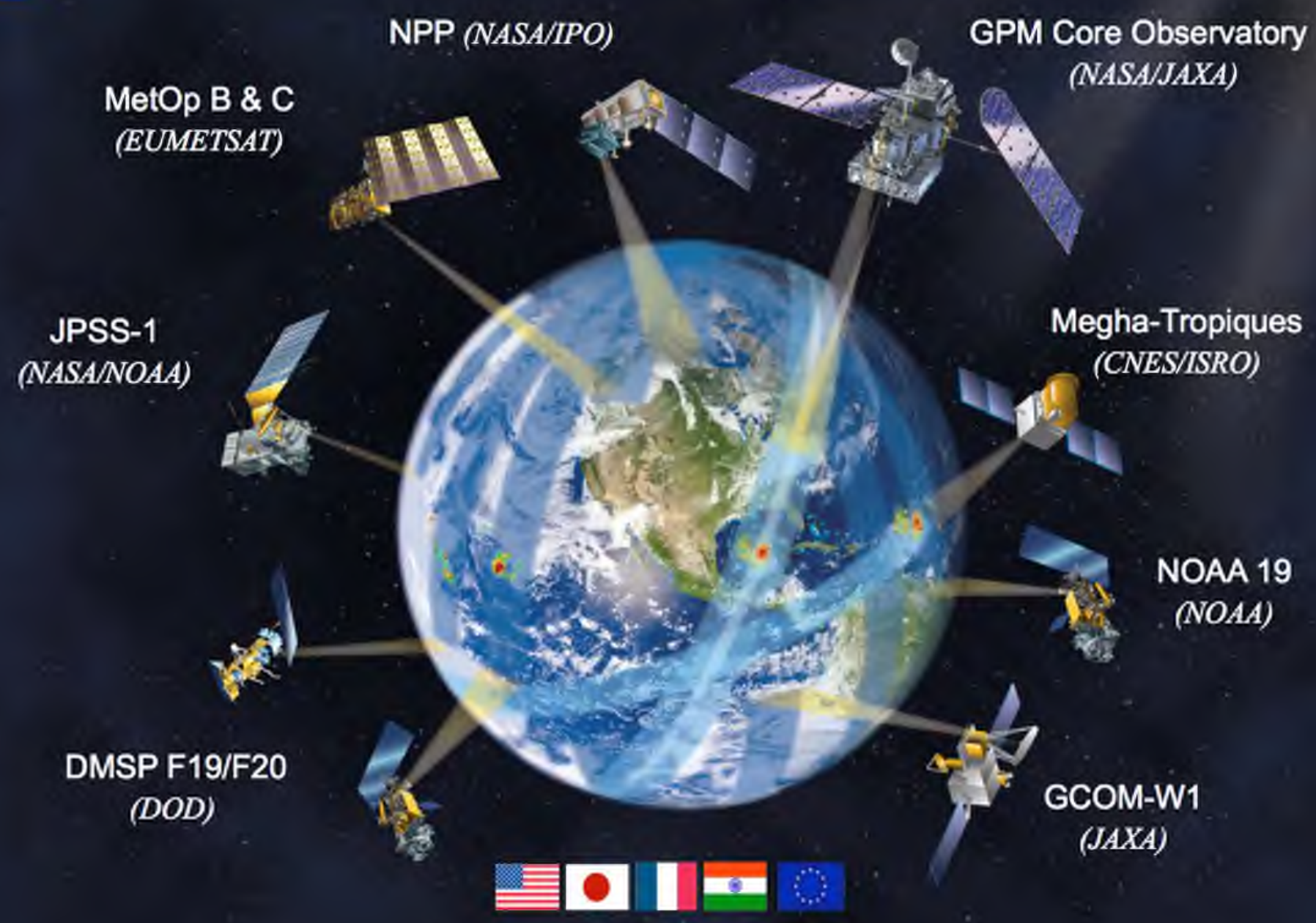




\section{Image Registration Challenges}

- Remote Sensing vs. Medical or Other Imagery

- Variety in the types of sensor data and the conditions of data acquisition

- Size of the data

- Lack of a known image model

- Lack of well-distributed "fiducial points" resulting in lack of algorithms validation

- Navigation Error

- Atmospheric and Cloud Interactions
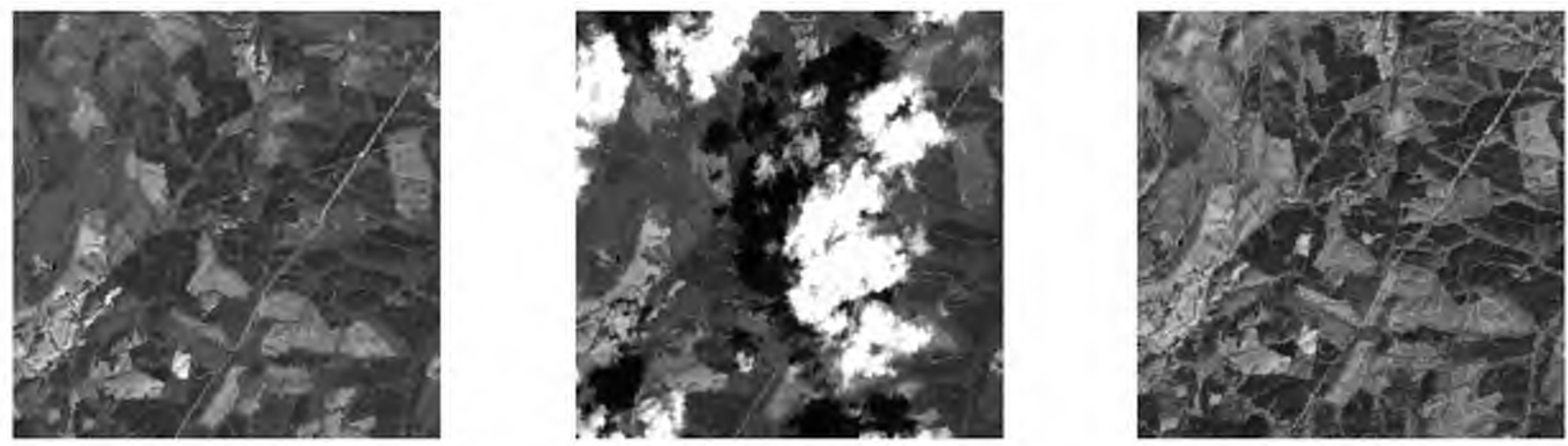

Three Landsat images over Virginia acquired in August, October, and November 1999 (Courtesy: Jeffrey Masek, NASA Goddard Space Flight Center) 


\section{Image Registration Challenges}

Atmospheric and Cloud Interactions

Atmospheric and Cloud Interactions Baja Peninsula, California; 4 different times of the day (GOES-8) (Reproduced from Le Moigne \& Eastman, 2005)

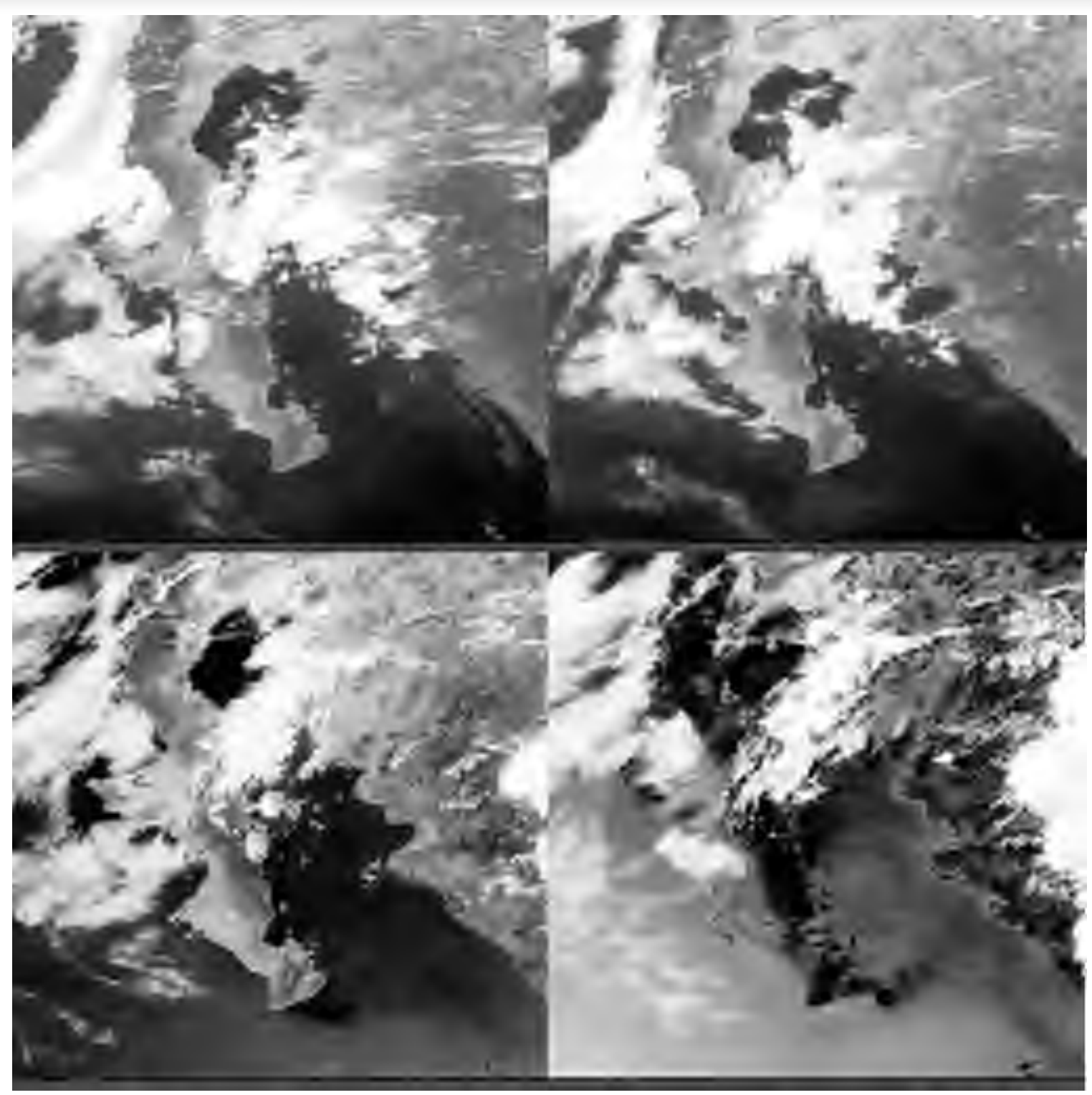




\section{Image Registration Challenges Multi-Temporal}

Mississippi and Ohio Rivers before \& after Flood of Spring 2002 (Terra/MODIS)

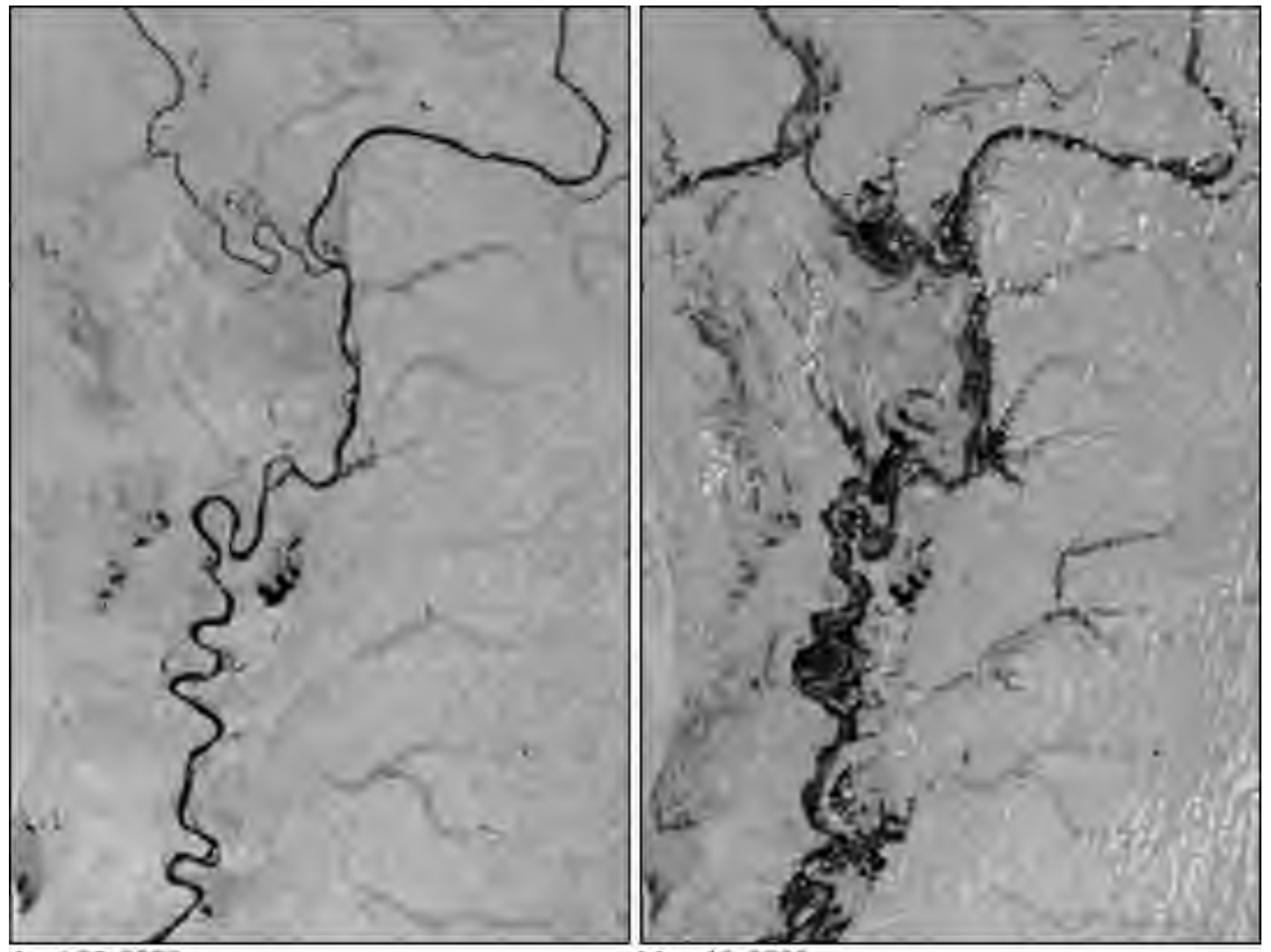




\section{What is Image Registration?}

- Image Registration/Feature-Based Precision Correction vs. Navigation or Model-Based Systematic Correction

1. Orbital, Attitude, Platform/Sensor Geometric Relationship, Sensor Characteristics, Earth Model, etc.

2. Navigation within a Few Pixels Accuracy

3. Image Registration Using Selected Features (or Control Points) to Refine Geo-Location Accuracy

- $\quad$ Mathematical Framework

$\circ \quad \mathrm{I} 1(\mathrm{x}, \mathrm{y})$ and $\mathrm{I} 2(\mathrm{x}, \mathrm{y})$ : images or image/map

$\checkmark \quad$ find the mapping $(f, g)$ which transforms I1 into I2: $\mathbf{I 2}(\mathbf{x}, \mathbf{y})=\mathbf{g}(\mathbf{I} 1(\mathbf{f x}(\mathbf{x}, \mathbf{y}), \mathbf{f y}(\mathbf{x}, \mathbf{y}))$

a. $\mathrm{f}$ : spatial mapping

b. g: radiometric mapping

- Spatial Transformations "f"

$\checkmark \quad$ Translation, Rigid, Affine, Projective, Perspective, Polynomial, ...

- Radiometric Transformations “g” (Resampling)

$\checkmark \quad$ Nearest Neighbor, Bilinear, Cubic Convolution, ...

- Algorithmic Framework (Brown, 1992)

1. Search Space of potential transformations

2. Feature Space of information extracted from the 2 datasets

3. Similarity Metric used to match the 2 sets of features

4. Search Strategy to find the optimal transformation

5. Resampling Method to create the corrected image

6. Validation Method to evaluate the accuracy of the registration 


\section{What is Image Registration?}

- Image Registration/Feature-Based Precision Correction vs. Navigation or Model-Based Systematic Correction

1. Orbital, Attitude, Platform/Sensor Geometric Relationship, Sensor Characteristics, Earth Model, etc.

2. Navigation within a Few Pixels Accuracy

3. Image Registration Using Selected Features (or Control Points) to Refine Geo-Location Accuracy

- $\quad$ Mathematical Framework

$\circ \quad \mathrm{I} 1(\mathrm{x}, \mathrm{y})$ and $\mathrm{I} 2(\mathrm{x}, \mathrm{y})$ : images or image/map

$\checkmark \quad$ find the mapping (f,g) which transforms I1 into I2: $\mathbf{I 2}(\mathbf{x}, \mathbf{y})=\mathbf{g}(\mathbf{I 1}(\mathbf{f x}(\mathbf{x}, \mathbf{y}), \mathbf{f y}(\mathbf{x}, \mathbf{y}))$

a. $\quad \mathrm{f}$ : spatial mapping

b. g: radiometric mapping

- Spatial Transformations "f"

$\checkmark \quad$ Translation, Rigid, Affine, Projective, Perspective, Polynomial, ...

- Radiometric Transformations “g” (Resampling)

$\checkmark$ Nearest Neighbor, Bilinear, Cubic Convolution, ...

- Algorithmic Framework (Brown, 1992)

1. Search Space of potential transformations

2. Feature Space of information extracted from the 2 datasets

3. Similarity Metric used to match the 2 sets of features

4. Search Strategy to find the optimal transformation

5. Resampling Method to create the corrected image

6. Validation Method to evaluate the accuracy of the registration 


\section{Operational Solutions The Landsat ETM+ Example}

- Sensor Knowledge

- Sensor geometry

- Sensor to platform

- Orbit

- Terrain data (DEM)

- Radiometric model

- Geodetic accuracy

- Database of GCPs derived from USGS data

- Normalized correlation

- Updates navigation models

- Results: RMSE $\sim 54 \mathrm{~m}$

- Band-to-band registration

- Selected tie-points in high-freq. arid regions

- Normalized correlation

- Subpixel by second order fit to $3 \times 3$ neighborhood

- Result: 0.1 to 0.2 subpixel
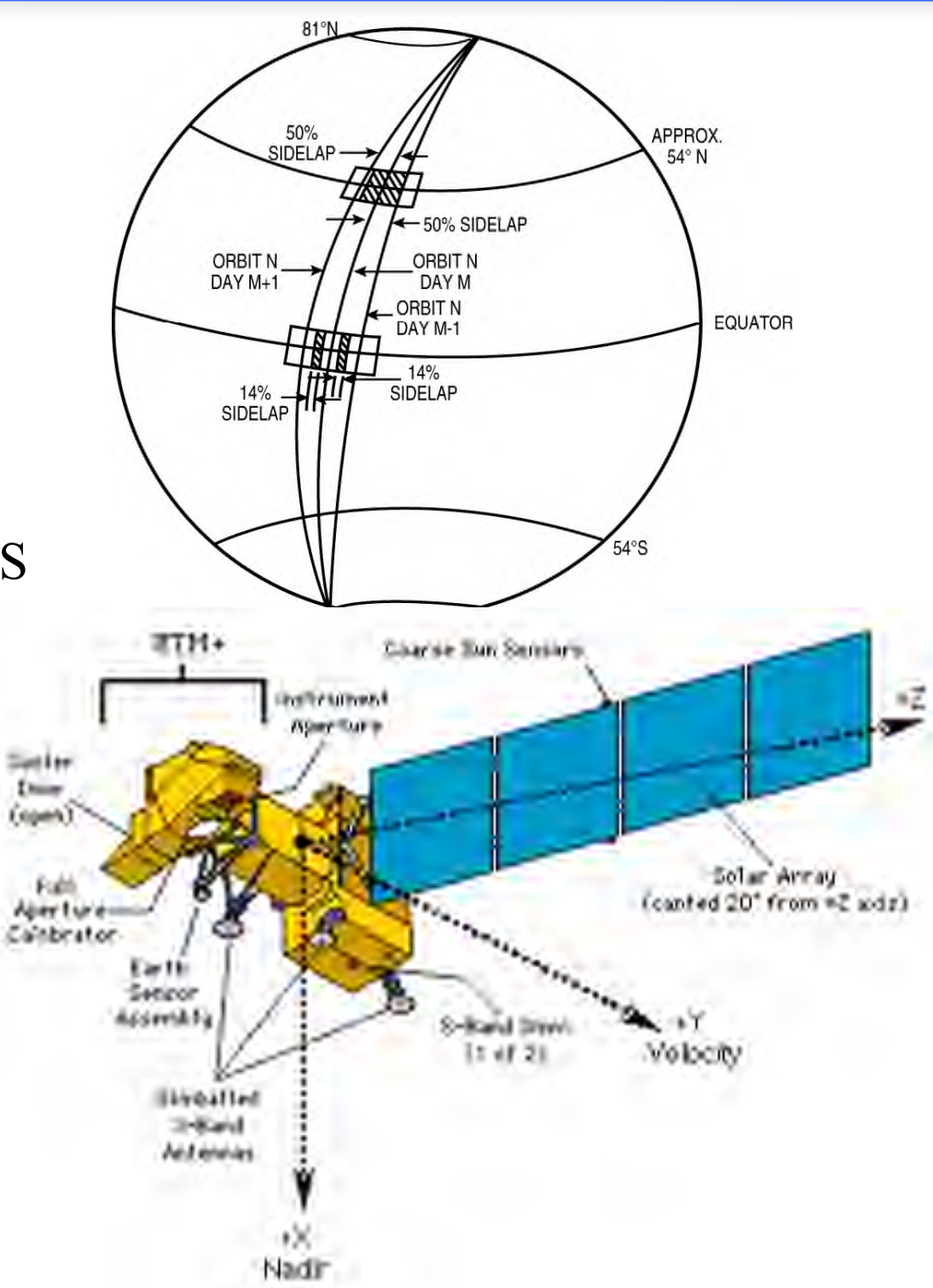


\section{Operational Solutions \\ Normalized Cross-Correlation (NCC) Often Used}

\begin{tabular}{|l|l|l|l|l|}
\hline Instrument & Satellite & Resolution & Similarity & Subpixel \\
\hline ASTER & Terra & $15 \mathrm{~m}-90 \mathrm{~m}$ & NCC w/ DEM & Fit to surface \\
\hline GOES & GOES I-M & $1 \mathrm{~km}-8 \mathrm{~km}$ & $\begin{array}{l}\text { NCC } \text { w/ vector } \\
\text { coastlines }\end{array}$ & $\begin{array}{l}\text { Bi-section } \\
\text { search }\end{array}$ \\
\hline MISR & Terra & $275 \mathrm{~m}$ & NCC w/ DEM & Least squares \\
\hline MODIS & Terra & $250 \mathrm{~m}-1 \mathrm{~km}$ & NCC w/ DEM & Fixed grid \\
\hline HRS & SPOT & $2.5 \mathrm{~m}$ & NCC w/ DEM & Not described \\
\hline ETM+ & Landsat-7 & $15 \mathrm{~m}-60 \mathrm{~m}$ & $\begin{array}{l}\text { NCC to arid } \\
\text { region CPS }\end{array}$ & Fit to surface \\
\hline VEGETATION & SPOT & $1 \mathrm{~km}$ & NCC w/ DEM & Not described \\
\hline
\end{tabular}




\section{Image Registration Algorithm Classifications}

- Area-Based vs. Feature-Based

- Often Combination of Area- and Feature-Based

- Alternate Classification:

- Manual Registration

- Correlation-Based Methods

- Fourier-Domain and Other Transform-Based Methods

- Mutual Information and Distribution-Based Approaches

- Feature-Point Methods

- Contour- and Region-Based Approaches 


\section{Feature Extraction}

\section{- Features:}

- Gray levels

- Salient points - Matched point-to-point or globally

- Edge or edge-like, e.g., Sobel, Canny

- Fourier coefficients

- Gabor, Wavelets, Directional Gabor or Wavelets, Shearlets, etc.

- Corners, e.g., Kearny, Harris and Stephens, Shi and Tomasi

- Lines (Hough and Generalized), Contours (Govindu et al), Regions (Region Segmentation, e.g., Tilton)

- Marked Point Processes (MPP): probabilistic framework with configuration space consisting of an unknown number of parametric objects

- Scale invariant feature transform (SIFT-Lowe) and variants, e.g., Speeded Up Robust Features (SURF)

- More recently, Neural Networks (NN) have been used for registration 


\section{Similarity Metrics}

\section{- Cross-correlation}

- Maximize cross-correlation over image overlap

$$
I_{1}(x, y) \circ I_{2}(x, y)=\sum_{m=0}^{M-1} \sum_{n=0}^{N-1} I_{1}(m, n) I_{2}(x+m, y+n)
$$

- Normalized cross-correlation (NCC)

- Maximize normalized cross-correlation

$$
N C C_{I_{1}, I_{2}}(x, y)=\frac{\sum_{m=0}^{M-1} \sum_{n=0}^{N-1}\left[I_{1}(m, n)-\bar{I}_{1}\right]\left[I_{2}(x+m, y+n)-\bar{I}_{2}\right]}{\sqrt{\sum_{m=0}^{M-1} \sum_{n=0}^{N-1}\left[I_{1}(m, n)-\bar{I}_{1}\right]^{2} \cdot \sum_{m=0}^{M-1} \sum_{n=0}^{N-1}\left[I_{2}(x+m, y+n)-\bar{I}_{2}\right]^{2}}}
$$

- Mutual information (MI):

Maximizes the degree of statistical dependence between the images

or using histograms, maximizes

$$
M I\left(I_{1}, I_{2}\right)=\sum_{g_{1}} \sum_{g_{2}} p_{I_{1}, I_{2}}\left(g_{1}, g_{2}\right) \cdot \log \left(\frac{p_{I_{1}, I_{2}}\left(g_{1}, g_{2}\right)}{p_{I_{1}}\left(g_{1}\right) \cdot p_{I_{2}}\left(g_{2}\right)}\right),
$$

$$
M I\left(I_{1}, I_{2}\right)=\frac{1}{M} \sum_{g_{1}} \sum_{g_{2}} h_{I_{1}, I_{2}}\left(g_{1}, g_{2}\right) \cdot \log \left(\frac{M h_{I_{1}, I_{2}}\left(g_{1}, g_{2}\right)}{h_{I_{1}}\left(g_{1}\right) \cdot h_{I_{2}}\left(g_{2}\right)}\right)
$$

where $M$ is the sum of all histogram entries, i.e., number of pixels (in overlapping subimage) 


\section{Similarity Metrics (cont.)}
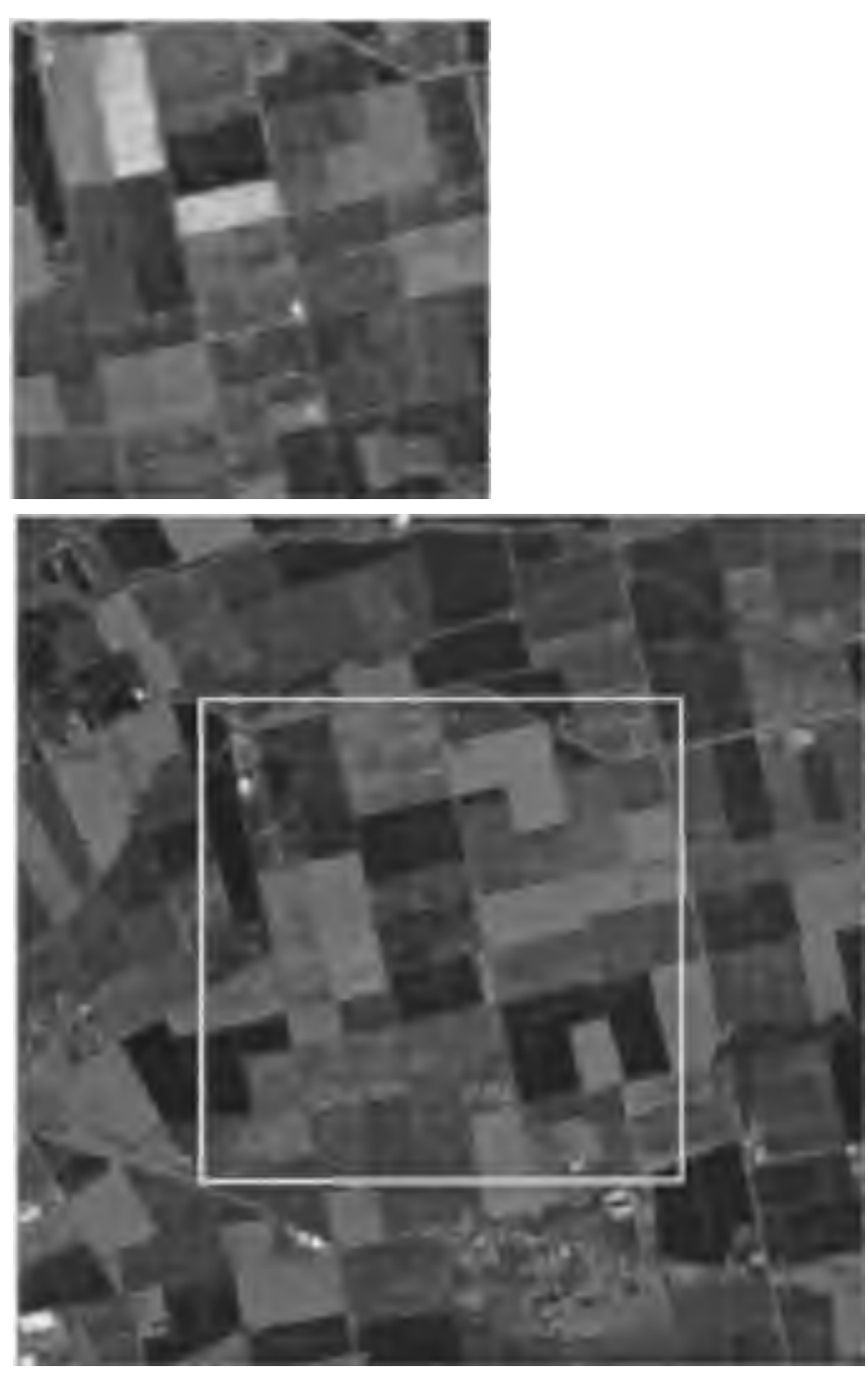
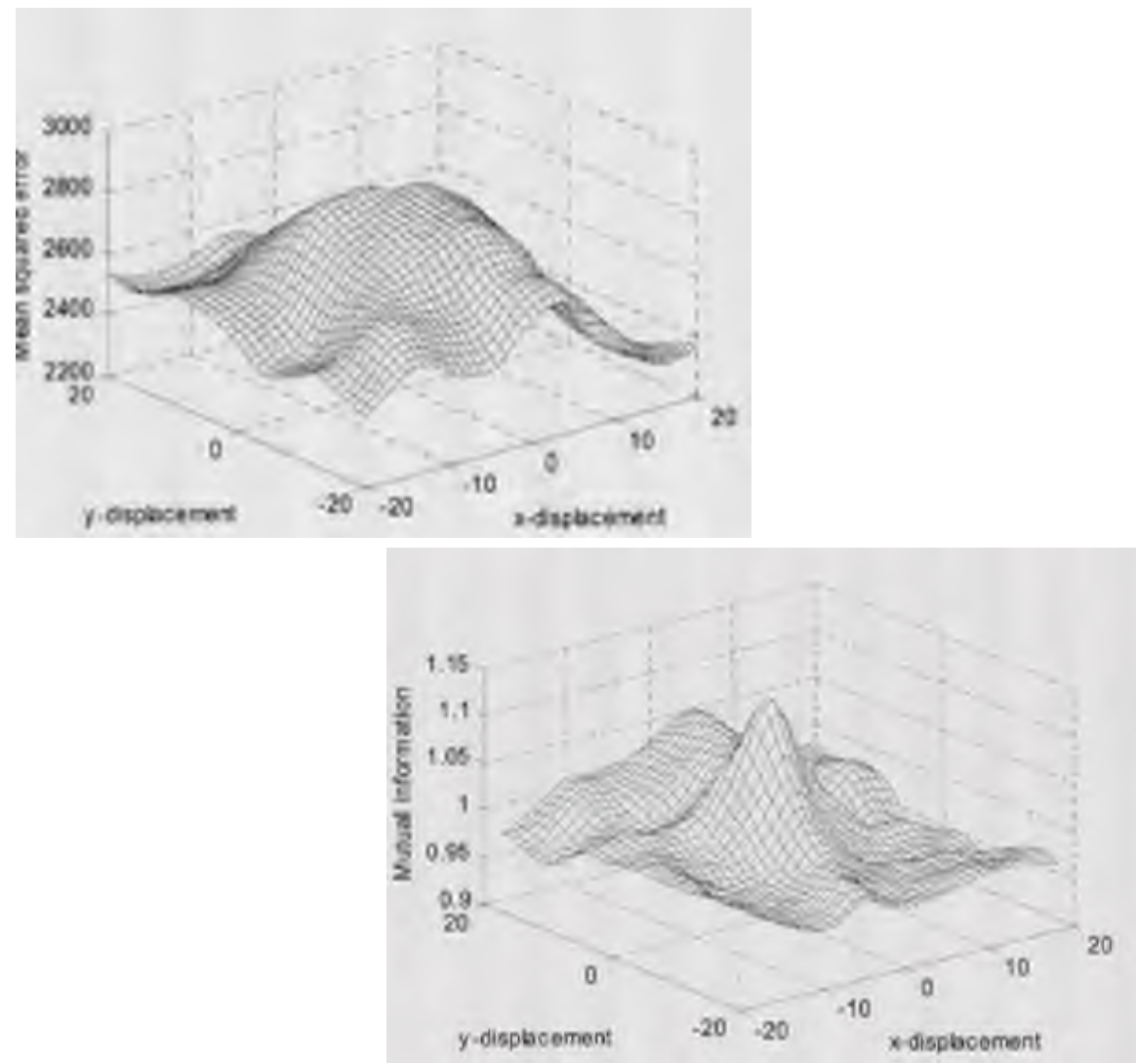

MI vs. $L_{2}$-norm and NCC applied to Landsat-5 images 


\section{Other Similarity Metrics}

- Partial Hausdorff distance (PHD):

$$
H_{K}\left(I_{1}, I_{2}\right)=K_{p_{1} \in I_{1}}^{\text {th }} \min _{p_{2} \in I_{2}} \operatorname{dist}\left(p_{1}, p_{2}\right),
$$

where $1 \leq K \leq\left|I_{1}\right|$ (Huttenlocher et al, Mount et al)

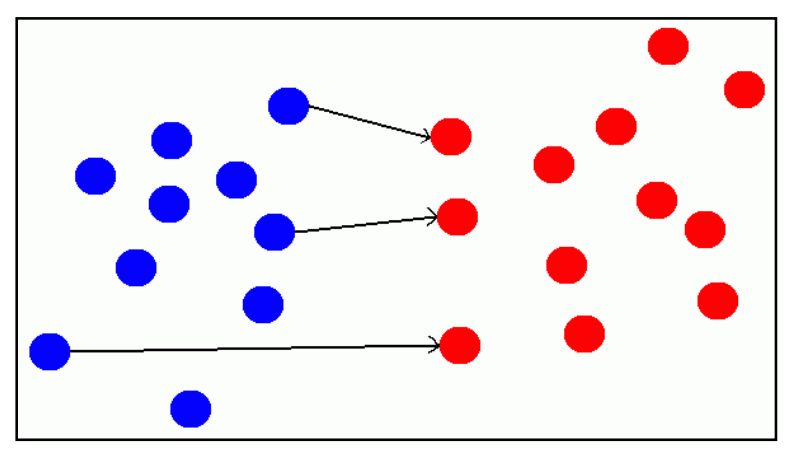

- Discrete Gaussian mismatch (DGM):

$$
\operatorname{DGM}_{\sigma}\left(I_{1}, I_{2}\right)=1-\frac{\sum_{a \in I_{1}} w_{\sigma}(a)}{\left|I_{1}\right|}
$$

where $w_{\sigma}(a)$ denotes the weight of point $a$, and $w_{\sigma}(a)=\exp \left(-\frac{\operatorname{dist}\left(a, I_{2}\right)^{2}}{2 \sigma^{2}}\right)$ 


\section{Image Matching Strategies}

- Matching strategies matched with feature extraction techniques

- Some methods:

- Exhaustive Search

- FFT/Phase Correlation - Fourier Mellin Transform

- Optimization:

- Steepest Gradient Descent

- Levenberg-Marquart

- Stocchastic Gradient

- Robust Feature Matching (RFM)

- Genetic algorithms (including binary shapes)

- Neural Networks (esp. for quantum \& cognitive computing)

- Global or local registration

- Various image representations, e.g., Multi-resolution and quadtrees 


\section{Some Recent Image Registration Results Wavelet and Wavelet-Like Based Algorithms}

Edge, Wavelet and Wavelet-Like Based Registration Framework

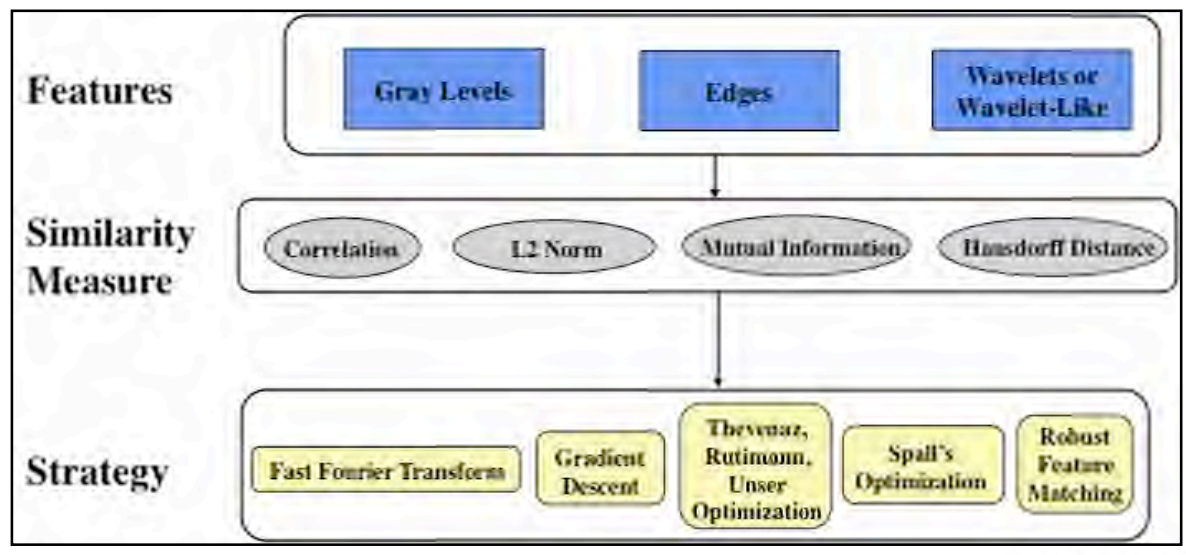

- Wavelets are fundamentally isotropic, i.e., no directional sensitivity

- Generalization of wavelets to be anisotropic $=>$ Shearlets, which refine the wavelet construction by including a directional component

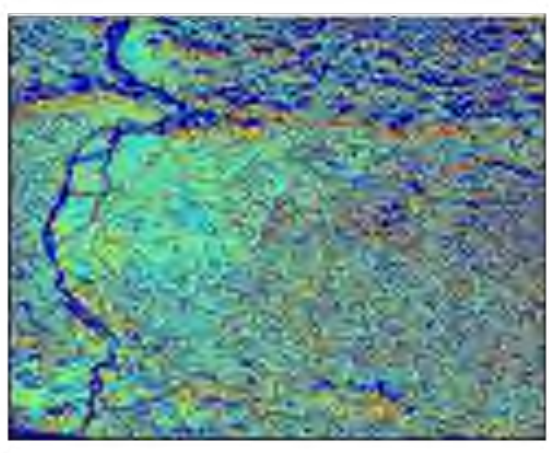

SAR image (1024x1024)
Wavelet Features
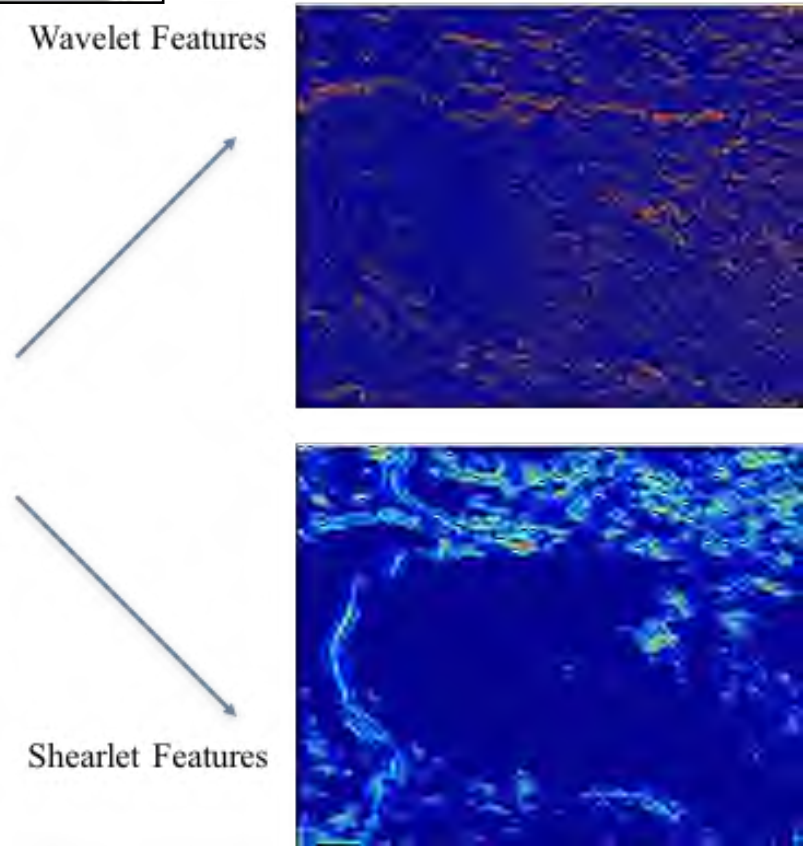


\section{Some Recent Image Registration Results Landsat Warped and Noise Experiments}

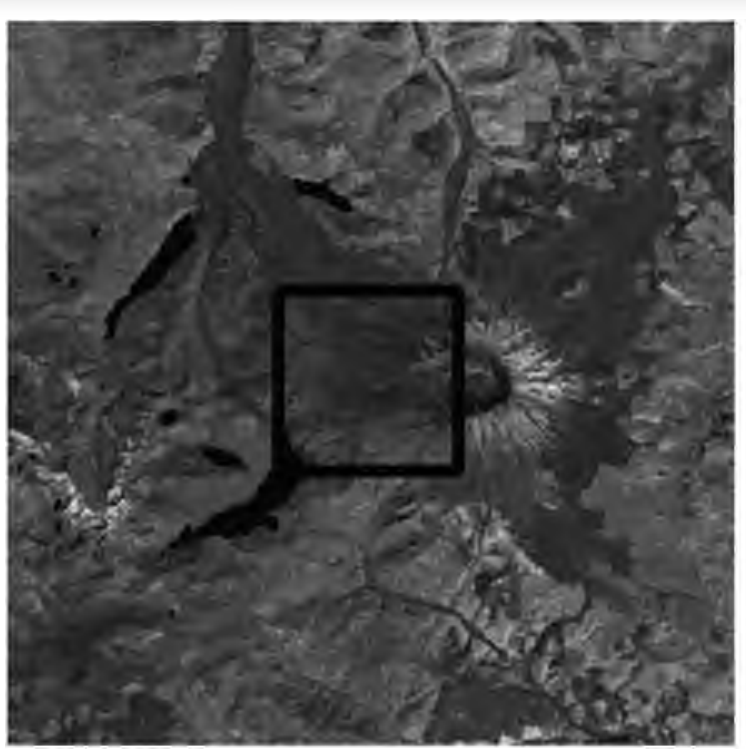

$256 \times 256$ Landsat-7 ETM+ images of

Washington, DC, (left) without and (right) with Gaussian noise added. The parameters for the noise are mean $\mu=$ 0 and variance $\sigma 2=0.05$

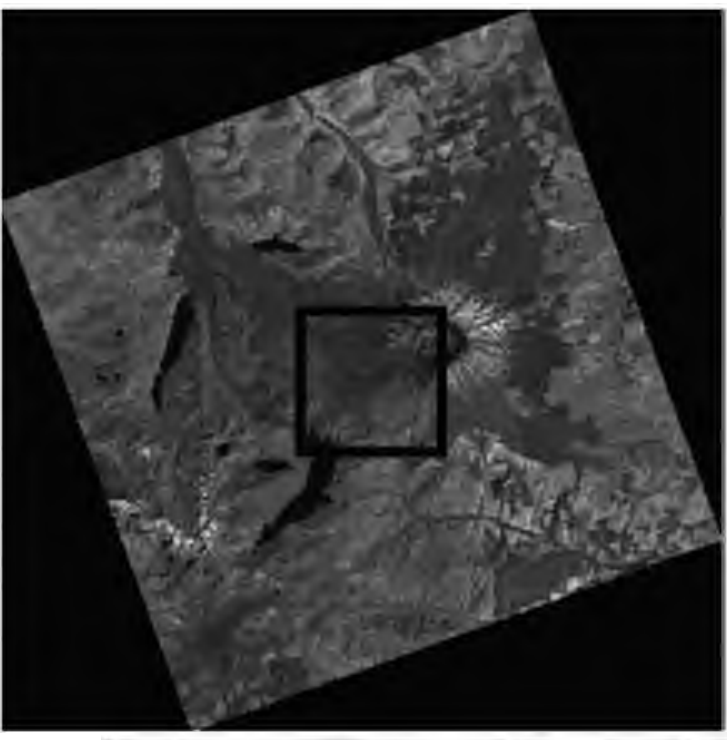

Geometrically warped synthetic input images. The full source image is $1024 \times 1024$ Landsat-5 TM image from the Mount Hood are. The extracted images are $256 \times 256$.
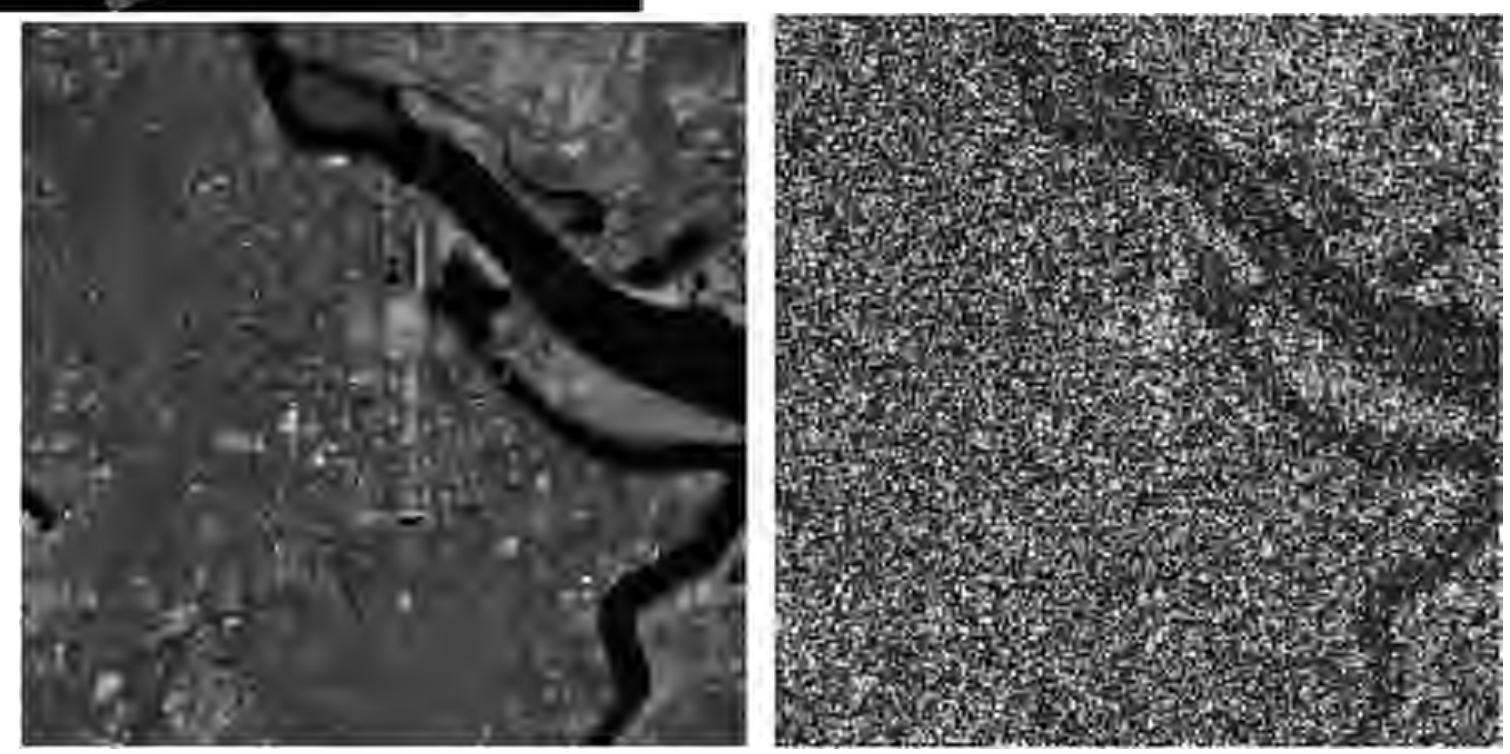
Shearlet-Based Registration Results As a Function of Warp

Comparison of Registration Algorithms for Landsat-TM Geometrically Warped Synthetic Experiments

\begin{tabular}{|c|c|c|c|c|c|}
\hline $\begin{array}{l}\text { Registration } \\
\text { Technique }\end{array}$ & $\begin{array}{l}\text { Number of } \\
\text { Coeverged } \\
\text { Experimets } \\
\text { (oul of } 200 \text { ) }\end{array}$ & $\begin{array}{l}\text { Percentage of } \\
\text { Consergod } \\
\text { Experiments }\end{array}$ & Mean RMSE & $\begin{array}{c}\text { Standard Deviation } \\
\text { RMSE }\end{array}$ & $\begin{array}{l}\text { Relative } \\
\text { Imaprovement }\end{array}$ \\
\hline $\begin{array}{l}\text { Spline } \\
\text { Wavelets }\end{array}$ & 108 & $5400 \%$ & .0019 & .0017 & - \\
\hline $\begin{array}{l}\text { Simoncelli } \\
\text { Band-Puss }\end{array}$ & 21 & $1050 \%$ & 0045 & 0014 & - \\
\hline $\begin{array}{l}\text { Simoncelli } \\
\text { Low-Pass }\end{array}$ & 113 & $55,50 \%$ & 0040 & .0036 & - \\
\hline Shearlets & 154 & $77.00 \%$ & 3.9513 & 1.5506 & - \\
\hline $\begin{array}{l}\text { Shearlett } \\
\text { Spline } \\
\text { Wavelete }\end{array}$ & 154 & $77.00 \%$ & .0058 & .0062 & $4259 \%$ \\
\hline $\begin{array}{l}\text { Shearlett } \\
\text { Sirmontelli } \\
\text { Band-Pass. }\end{array}$ & 154 & $77.00 \%$ & .0080 & .0050 & $633,33 \%$ \\
\hline $\begin{array}{l}\text { Shourlet + } \\
\text { Simonoeliti } \\
\text { Lom.Pass }\end{array}$ & 154 & $73.00 \%$ & ores & .0081 & $3628 \%$ \\
\hline
\end{tabular}




\section{Shearlet-Based Registration Results As a Function of Noise}

Comparison of Registration Algorithms for Noisy Landsat-ETM+ Synthetic Experiments $($ Variance $=0.05)$

\begin{tabular}{|c|c|c|c|c|c|}
\hline $\begin{array}{l}\text { Registration } \\
\text { Technique }\end{array}$ & $\begin{array}{l}\text { Number of } \\
\text { Converged } \\
\text { Experiments } \\
\text { (out of } 201 \text { ) }\end{array}$ & $\begin{array}{l}\text { Peroentage of } \\
\text { Comerged } \\
\text { Expenments }\end{array}$ & Mena RMSE & $\begin{array}{c}\text { Standard Deviation } \\
\text { RMSE }\end{array}$ & $\begin{array}{l}\text { Relative } \\
\text { Improvemen }\end{array}$ \\
\hline $\begin{array}{l}\text { Spline } \\
\text { Wavelets }\end{array}$ & 31 & $15.42 \%$ & (1579 & $x(x) 1$ & - \\
\hline $\begin{array}{l}\text { Simoncelli } \\
\text { Band-Pass }\end{array}$ & 42 & $2090 \%$ & .0905 & $\sim 0$ & - \\
\hline $\begin{array}{l}\text { Simoncelli } \\
\text { Low-Pass }\end{array}$ & 67 & $33.33 \%$ & .0560 & $\sim 0$ & - \\
\hline Shearleis & 98 & $48.76 \%$ & 1.8486 & 1.1933 & - \\
\hline $\begin{array}{l}\text { Sheardet+ } \\
\text { Spline } \\
\text { Wavelets }\end{array}$ & 98 & $48.76 \%$ & .0468 & $\sim 0$ & $216.13 \%$ \\
\hline $\begin{array}{l}\text { Shearkit } \\
\text { Sirtonotlit } \\
\text { Based-Pats }\end{array}$ & 98 & $48.76 \%$ & .0505 & $\sim 0$ & $333.33 \%$ \\
\hline $\begin{array}{l}\text { Shearlet + } \\
\text { Simoncelli } \\
\text { Low-Pass }\end{array}$ & 99 & $48.76 \%$ & .0560 & $\sim 0$ & $46.27 \%$ \\
\hline
\end{tabular}




\section{Shearlet-Based Registration Results Multimodal Experiments}
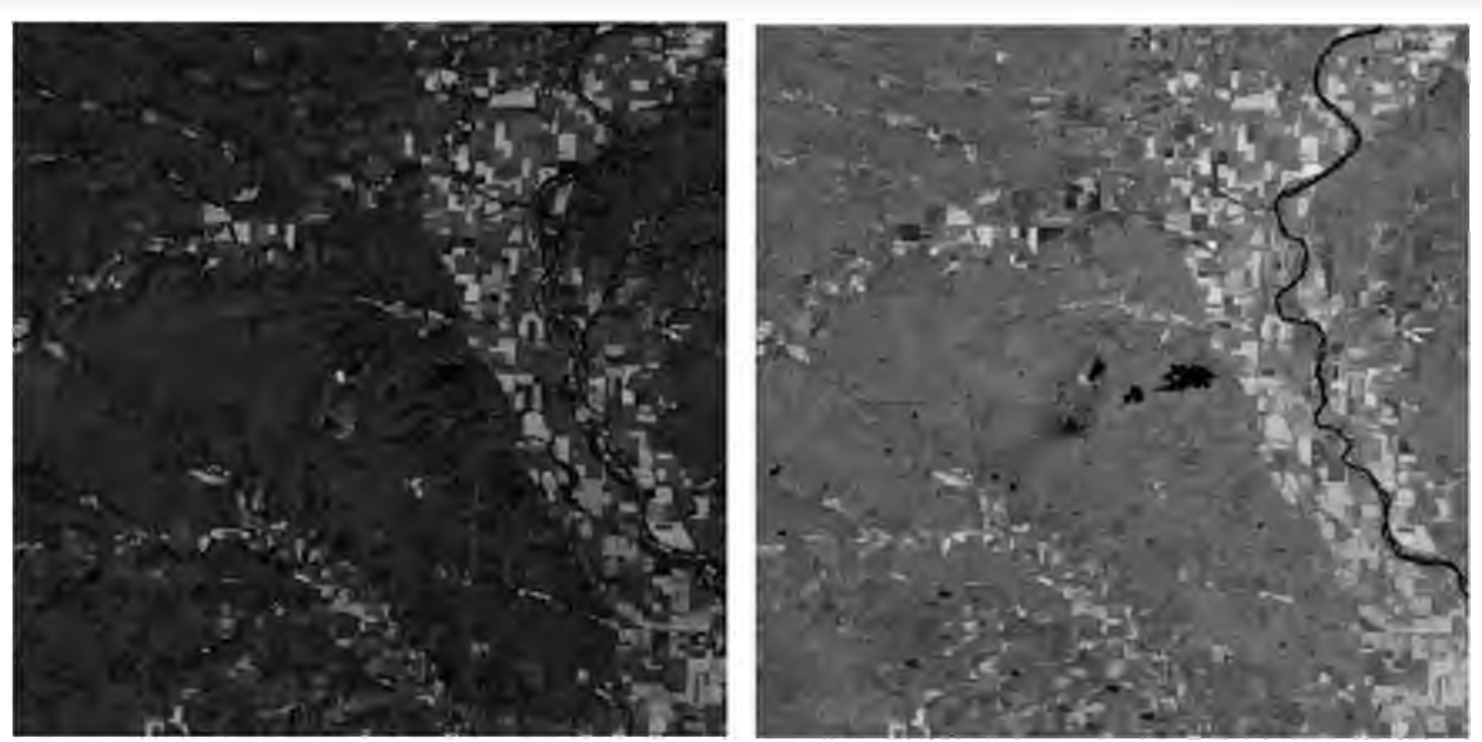

$1024 \times 1024$ images of (left) ETM+ Infared/Red band and (right) Near-Infared/NIR band of the Konza Prairie

Pixels computed by SIFT in the LIDAR shaded-relief (left) and optical (right) images of Washington State, connected by line segments. Note the lack of correspondence; such points are unsuitable for a registration algorithm.

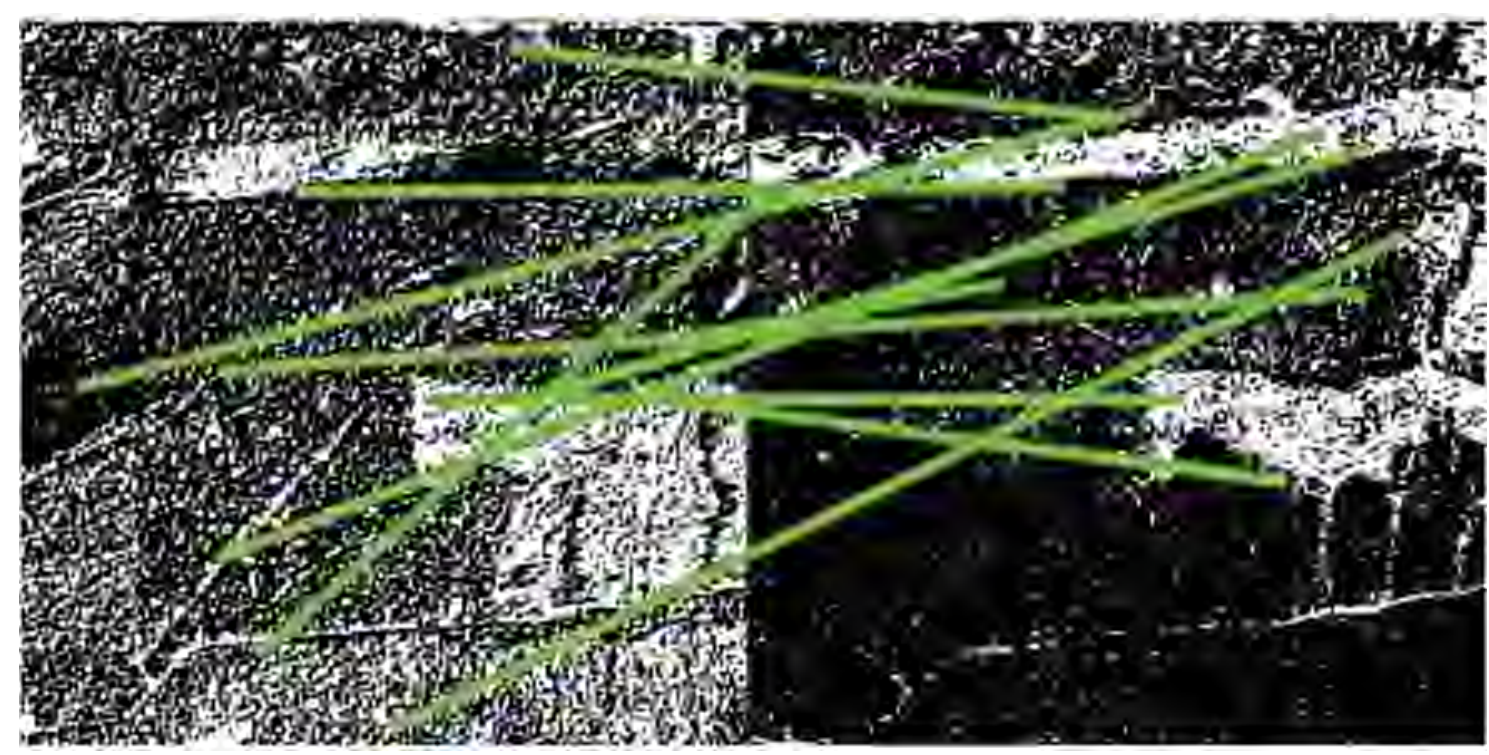




\section{Shearlet-Based Registration Results For LIDAR Data}

Comparison of Registration Algorithms for LIDAR Warped Synthetic Experiments

\begin{tabular}{|c|c|c|c|c|c|}
\hline $\begin{array}{l}\text { Registration } \\
\text { Teclmique }\end{array}$ & $\begin{array}{l}\text { Number of } \\
\text { Coeverged } \\
\text { Experimets } \\
\text { (out of } 201 \text { ) }\end{array}$ & $\begin{array}{l}\text { Percentage of } \\
\text { Coniergod } \\
\text { Expeniments }\end{array}$ & Meas RMSE & $\begin{array}{c}\text { Standard Deviation } \\
\text { RMSE }\end{array}$ & $\begin{array}{l}\text { Relative } \\
\text { Inprovemen! }\end{array}$ \\
\hline $\begin{array}{l}\text { Spline } \\
\text { Wavelets }\end{array}$ & 74 & $3682 \%$ & 3552 & .0256 & - \\
\hline $\begin{array}{l}\text { Simcocelli } \\
\text { Band.PAsss }\end{array}$ & 42 & $2090 \%$ & .0074 & $\sim 0$ & - \\
\hline $\begin{array}{l}\text { Simorscelli } \\
\text { Lou-Pass }\end{array}$ & 72 & $3582 \%$ & 2412 & .0166 & - \\
\hline Shearlets & 108 & $5373 \%$ & 01304 & .0012 & - \\
\hline $\begin{array}{l}\text { Shearlett } \\
\text { Spline } \\
\text { Wavelets }\end{array}$ & 111 & $5522 \%$ & 3222 & .0143 & $50.00 \%$ \\
\hline $\begin{array}{l}\text { Shearled } \\
\text { Simonicelti } \\
\text { Band-Pass }\end{array}$ & 108 & $53.73 \%$ & .0075 & $\sim 0$ & $157.14 \%$ \\
\hline $\begin{array}{l}\text { Shearlet + } \\
\text { Simorecelli } \\
\text { Lom-Pues }\end{array}$ & 111 & $5522 \%$ & .2432 & $\sim 0$ & $5471 \%$ \\
\hline
\end{tabular}




\section{Shearlet-Based Registration Results Multimodal Experiments}

Comparison of Registration Algorithms for ETM+ Infrared to NIR Multimodal Experiments

\begin{tabular}{|c|c|c|c|c|c|}
\hline $\begin{array}{l}\text { Registration } \\
\text { Technique }\end{array}$ & $\begin{array}{l}\text { Number of } \\
\text { Comerged } \\
\text { Experimenls } \\
\text { (out of 41) }\end{array}$ & $\begin{array}{l}\text { Percentage of } \\
\text { Converged } \\
\text { Expenments }\end{array}$ & Mcan RMSE & $\begin{array}{c}\text { Standard Deviation } \\
\text { RMSE }\end{array}$ & $\begin{array}{l}\text { Relative } \\
\text { Improvement }\end{array}$ \\
\hline $\begin{array}{l}\text { Spline } \\
\text { Wavelets }\end{array}$ & 25 & $60.98 \%$ & 2389 & 0137 & - \\
\hline $\begin{array}{l}\text { Simoncelli } \\
\text { Band-Pass }\end{array}$ & 18 & $43.90 \%$ & 2492 & $\sim 0$ & - \\
\hline $\begin{array}{l}\text { Simoncelli } \\
\text { Low-Pass }\end{array}$ & 34 & $82.93 \%$ & 2100 & $\sim 0$ & - \\
\hline Shearlets & 38 & $92.68 \%$ & 6678 & 3917 & $52.00 \%$ \\
\hline $\begin{array}{l}\text { Shearlet+ } \\
\text { Spline } \\
\text { Wavelets }\end{array}$ & 38 & $92.68 \%$ & 2465 & 0336 & $111.11 \%$ \\
\hline $\begin{array}{l}\text { Shearlet+ } \\
\text { Simoncelli } \\
\text { Band-Pass }\end{array}$ & 38 & $92.68 \%$ & 2492 & $\sim 0$ & $11.76 \%$ \\
\hline $\begin{array}{l}\text { Shearlet }+ \\
\text { Simoncelli } \\
\text { Low-Pass }\end{array}$ & 38 & $92,68 \%$ & 2100 & $\sim 0$ & \\
\hline
\end{tabular}




\section{Shearlet-Based Registration Results Multimodal Experiments (cont.)}

Comparison of Registration Algorithms for LIDAR to Optical Multimodal Experiments

\begin{tabular}{|c|c|c|c|c|c|}
\hline $\begin{array}{l}\text { Registration } \\
\text { Tectuniqpe }\end{array}$ & $\begin{array}{l}\text { Nember of } \\
\text { Converged } \\
\text { Experiments } \\
\text { (cilt of 101) }\end{array}$ & $\begin{array}{l}\text { Percentage of } \\
\text { Coasergod } \\
\text { Experiments }\end{array}$ & Mean RMSE & $\begin{array}{c}\text { Standard Deviation } \\
\text { RMSE }\end{array}$ & $\begin{array}{l}\text { Relatrie } \\
\text { Improvement }\end{array}$ \\
\hline $\begin{array}{l}\text { Spline } \\
\text { Wavelets }\end{array}$ & 55 & $54.46 \%$ & 3.4499 & .0012 & - \\
\hline $\begin{array}{l}\text { Simoncelli } \\
\text { Band-Pass }\end{array}$ & 61 & $6040 \%$ & 36542 & 0174 & - \\
\hline $\begin{array}{l}\text { Simoncelli } \\
\text { Low-Pass }\end{array}$ & 86 & $85.15 \%$ & 35918 & .0066 & - \\
\hline Shearlets & +4 & $87.13 \%$ & 15,6428 & 6.1668 & - \\
\hline $\begin{array}{l}\text { Shearle1 + } \\
\text { Spline } \\
\text { Wavelets }\end{array}$ & $\theta 0$ & $59,41 \%$ & 3.4222 & $\sim 0$ & $9.09 \%$ \\
\hline $\begin{array}{l}\text { Shearlet + } \\
\text { Simoncell, } \\
\text { Band-Pass }\end{array}$ & 65 & $6436 \%$ & 36518 & .0174 & $6.56 \%$ \\
\hline $\begin{array}{l}\text { Shearlet + } \\
\text { Simoncellit } \\
\text { Low-Pass }\end{array}$ & 88 & 87.135 & 35912 & .0083 & $2.33 \%$ \\
\hline
\end{tabular}




\section{Preliminary Image Registration Results Using Artificial Neural Networks}

Feed-forward neural network (FF-NN) for subpixel accuracy

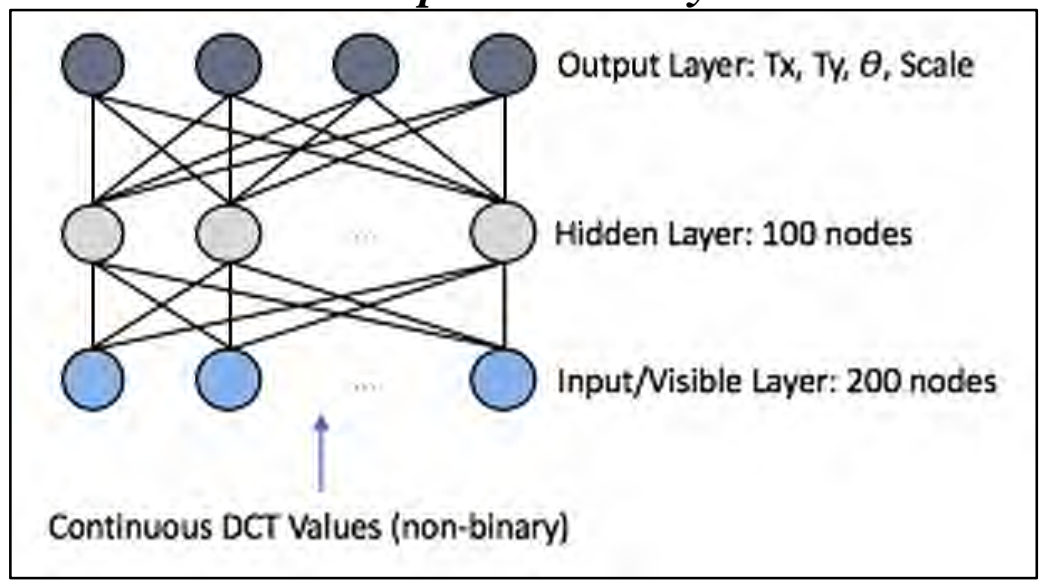

- Using Discrete Cosine Transform (DCT) coefficients as input provides subpixel accuracy

- Input: 100 DCT coefficients from reference image +100 DCT coefficients from test image

- Output: Transformation Variables (Tx, Ty, $\Theta, \mathrm{s})$

- Score: Subpixel registration accuracy if mean RMS error $<1.0$ per pixel

- Subpixel accuracy on 50\% of the test images in $<500$ training epochs. Running for longer increases accuracy

- Training set must be large enough to capture the range of values for rotation/translation in the test set

- Training set of 100 images randomly rotated/translated from a source image is enough to learn: $+/-45^{\circ}$ rotation coupled with $+/-10$ pixels translation

- Training set of 300 images is enough to learn:

$+/$ - 120 을 rotation, no translation

+/- 80 pixels translation, no rotation

- Current experiments using Deep Belief Networks and Restricted Boltzman Machines 


\section{Conclusions}

- Brief introduction to remote sensing image registration and its main components:

- Feature Extraction

- Similarity Metrics

- Search Strategies

- Components combined appropriately and adapted to:

- Type of data (e.g., edge- vs. texture-rich)

- Size of data and computational resource needed

- Required accuracy

- Initial conditions

- Future Work:

- Systematic assessment of various algorithms

- Creating benchmark datasets 